
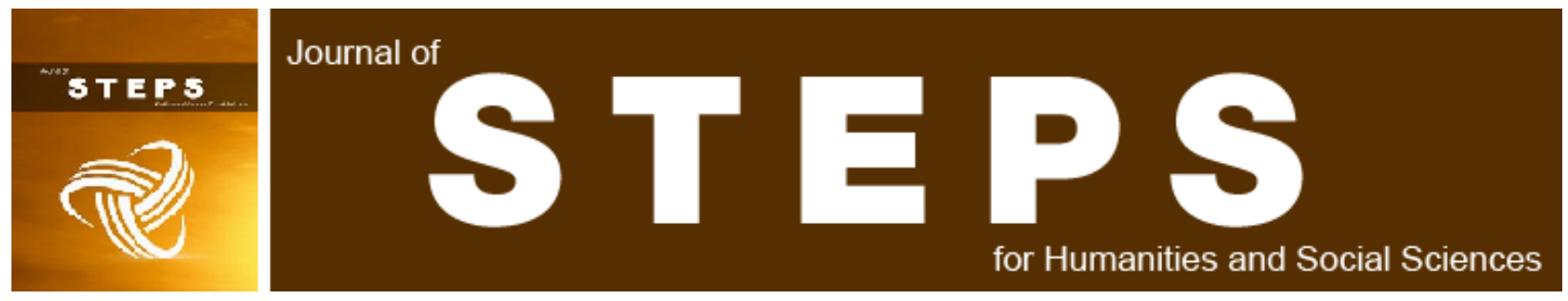

Volume 1 | Issue 1

Article 5

\title{
Service properties in the cities of Alhayi and Azizia
}

Ayat Allaami

Wasit University, Iraq, Aylul_2021@gmail.com

Sabeeh Farhan

Wasit University, Iraq

Shakir Alzamly

Wasit University, Iraq

Follow this and additional works at: https://www.steps-journal.com/jshss

Part of the Education Commons

\section{Recommended Citation}

Allaami, Ayat; Farhan, Sabeeh; and Alzamly, Shakir (2022) "Service properties in the cities of Alhayi and Azizia," Journal of STEPS for Humanities and Social Sciences: Vol. 1 : Iss. 1 , Article 5.

Available at: https://doi.org/10.55384/2790-4237.1001

This Original Study is brought to you for free and open access by Journal of STEPS for Humanities and Social Sciences (STEPS). It has been accepted for inclusion in Journal of STEPS for Humanities and Social Sciences by an authorized editor of Journal of STEPS for Humanities and Social Sciences (STEPS). 


\title{
الخصائص الخدمية في مدينتي الحي والعزيزية
}

\author{
* آيات حاكم وحيد اللامي د.شاكر مسير لفته الز امل
}

المستخلص

لا يعد توفر خدمات البنى التحتية في منطقة ما كافيا بل يجب أَنّ تتمتع بالكفاءة العالية فهي مهمه للسكان، لأنة فالسكن بالمفهوم الحديث لا يعد صالحا بدون منظومة خدمية متكاملة، لأن توفر الخدان الخدات في في المدينة يحقق

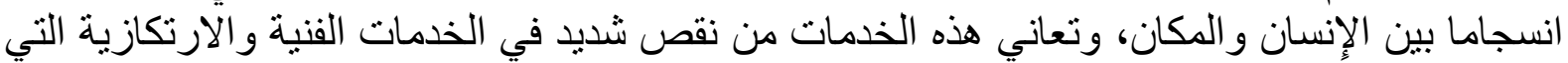

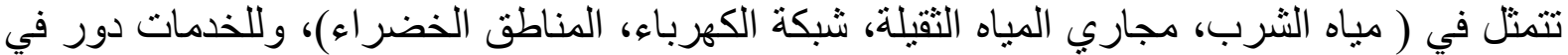

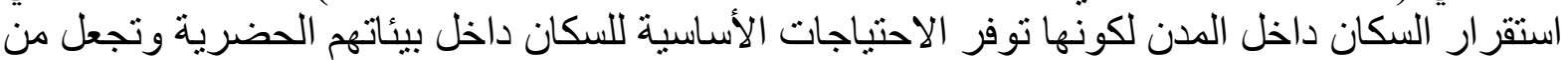

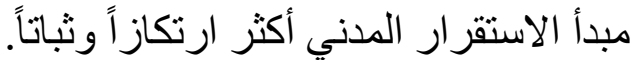

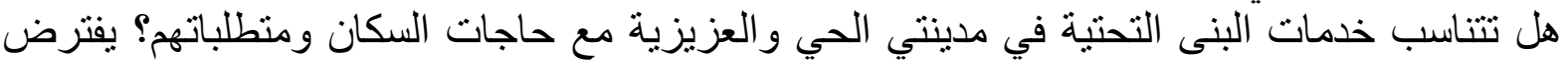

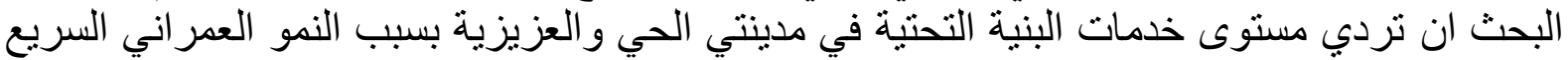
وقصور عمليات التخطيط لقطاع الخدمات. يهذف البحث الى در اسة الخدمات في مدينتي الحي و العزيزية، مسلطين الضوء التهات على الخدمات الاساسية في المدينتين المتمثلة بخدمات الماء و الصرف الصي في الصحي الكهرباء و النفايات الصلبة.

كلمات مفتاحية: خدمات، مناطق خضر اء، خدمات الكهرباء.

\section{Service properties in the cities of Alhayi and Azizia}


*Ayat Hakim Allaami, College of Education for Human Sciences, Wasit University, Iraq. Shakir Masir Alzamly, College of Education for Human Sciences, Wasit University, Iraq.

\begin{abstract}
The availability of infrastructure services in an area is not sufficient, but rather it must be highly efficient, as it is important for the population. Housing in the modern sense is not valid without an integrated service system, because the availability of services in the city achieves harmony between the person and the place, and these services suffer from a severe lack of services Technical and subsidiaries represented in (drinking water, heavy water courses, electricity network, green areas), and services have a role in stabilizing the population within cities because they provide the basic needs of the population within their urban environments and make the principle of civil stability more anchored and stable.
\end{abstract}

Keywords: Services, green areas, electricity services. 
تقع مدينة الحي بين خطي طول (45,43 -.46,18) شرقا، وبين دائرتي عرض (56، 31-25،32) شمالا، اما

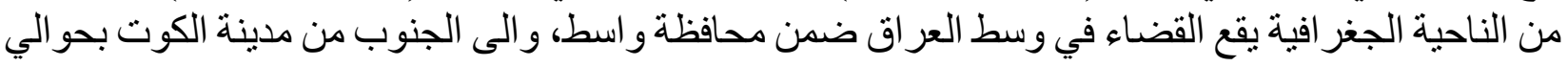

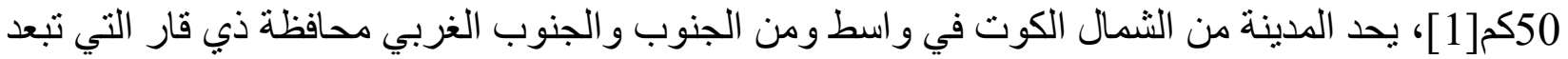
عنها بمسافة 30كم، ومحافظة القادسية، اما من الغرب تحدها النعمانية ومن جهة الثرق و الثمال الشرقي ناحية و اسط]

اما مدينة العزيزية و هي احدى مدن محافظة واسط تقع جنوب شرق مدينة بغداد وتبعد عنها مسافة 85كم و ون

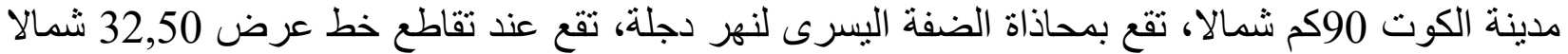

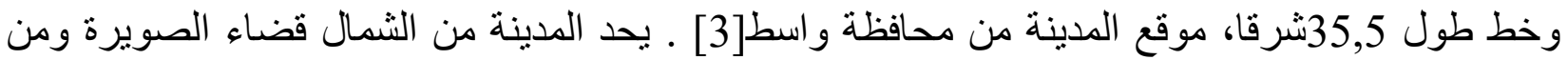

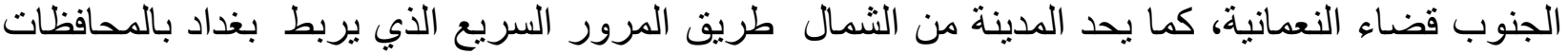

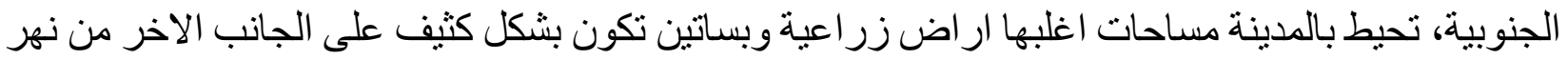

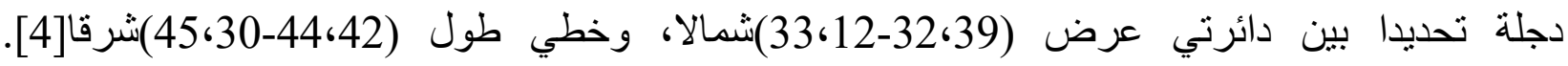

خريطة(1).

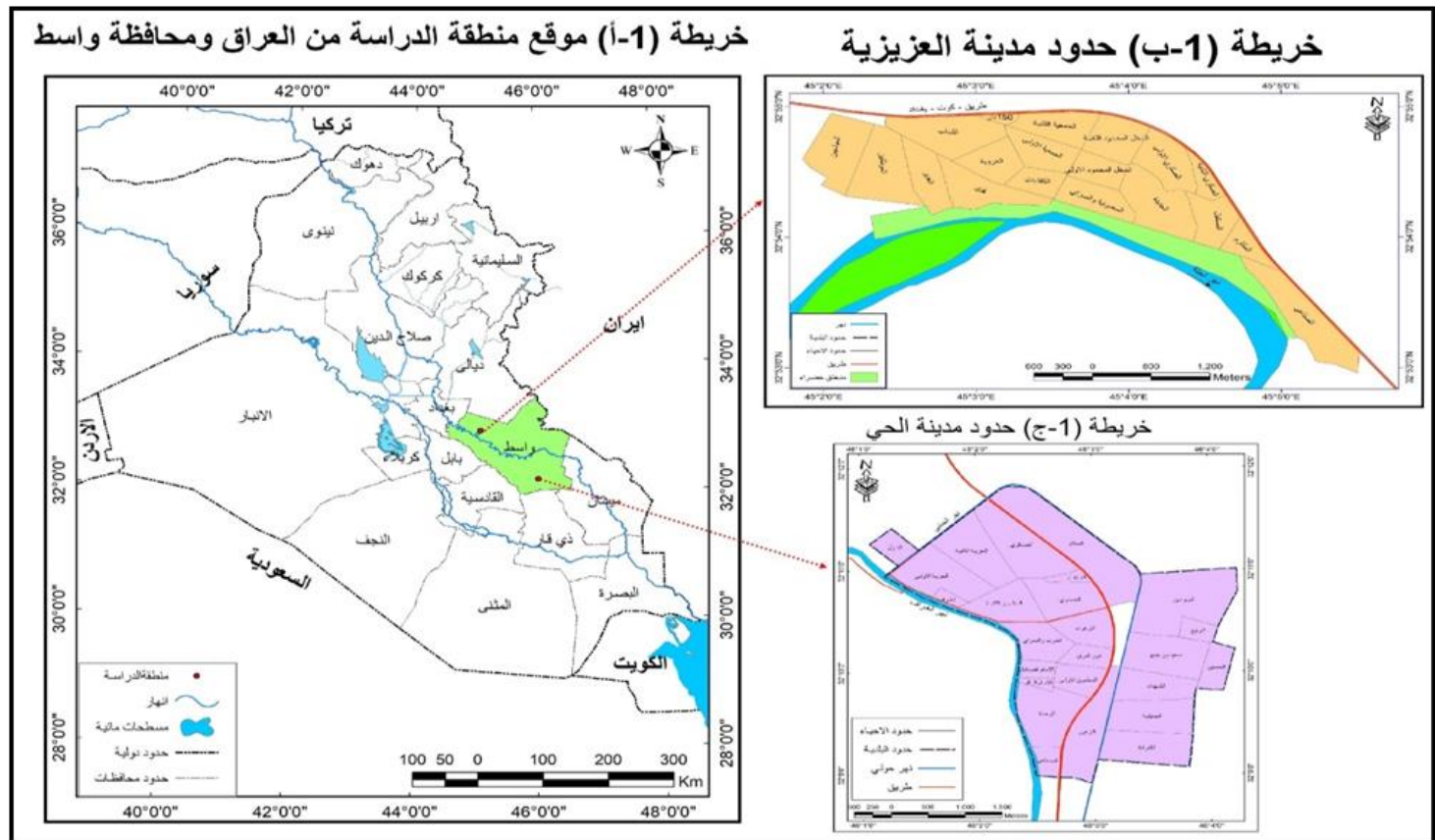

خريطة (1): موقع مدينتي الحي و العزيزية بالنسبة للعر اق و لمحافظة واسط لعام 2020, المصدر : وزارة الموارد المائية الهيئة العامة للمساحة، خريطة العر اق الادارية، مقياس 1:1000000 لسنة 2010، مديرية بلديات الحي و العزيزية، شعبة GIS.

خدمات البنية التحتية المياه الصالحة للشرب

يعد في مدينة العزيزية نهر دجلة المصدر الرئيس للمياه الذي يقطع المدينة من الثمال الى الجنوب الذي تعتمد

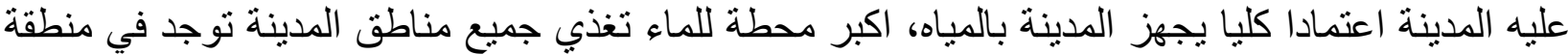

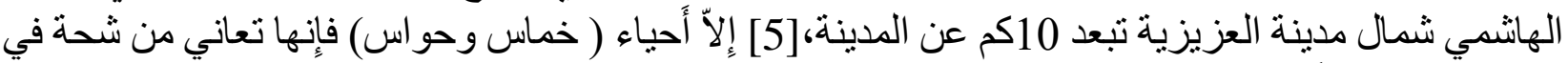

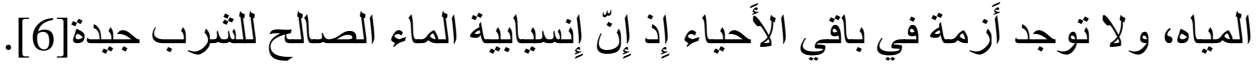
وبلغ عدد مشاريع الماء(3) و (15) مجمع 3منها داخل المدينة، أَولاً مشروع ماء العزيزية الموحد تأسس عام

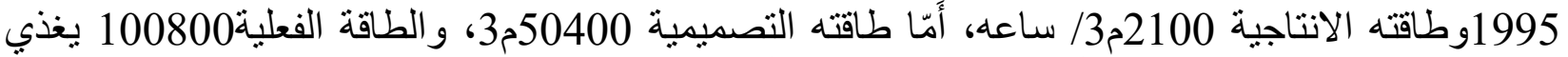

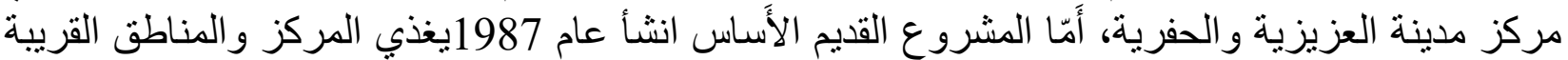

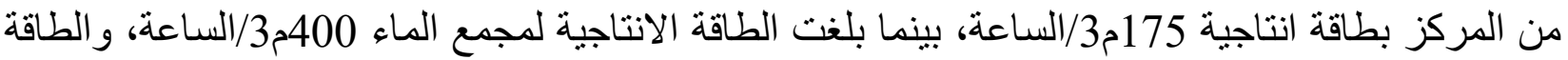




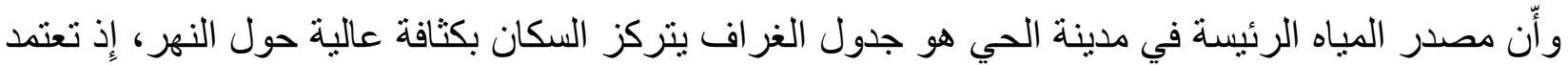

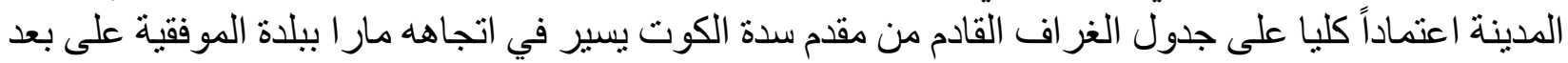

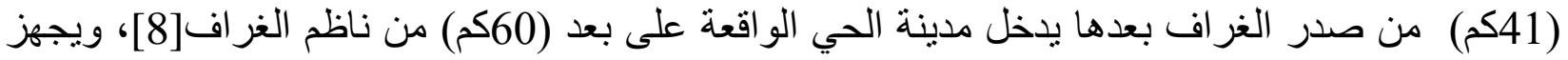

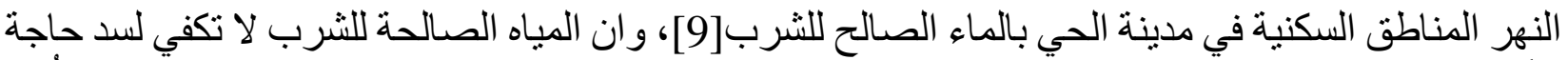

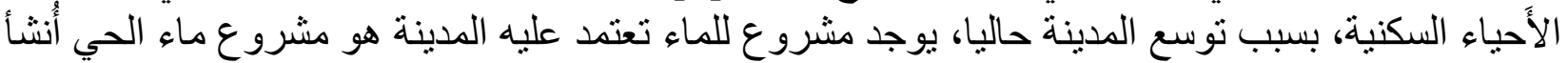

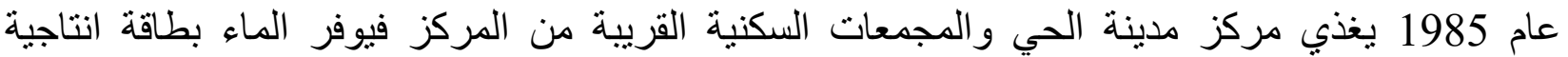

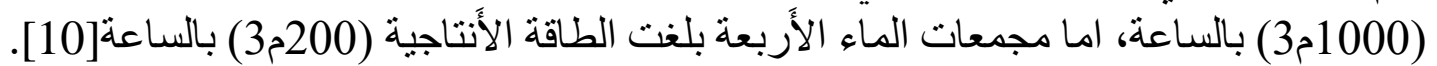

ان افتقار الانابيب الناقلة للماء لأعمال الصيانة وقدمها وصغر اقطار ها ولا سيما في المناطق السكنية القديمة

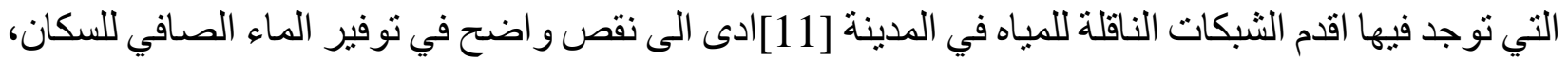
تعاني حي الحرية من شحة المياه في الصيف بسبب قدم الانابيب الناقلة للمياه وعدم كفاءتها اثر على نو عية

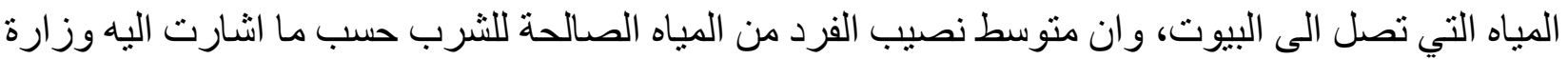

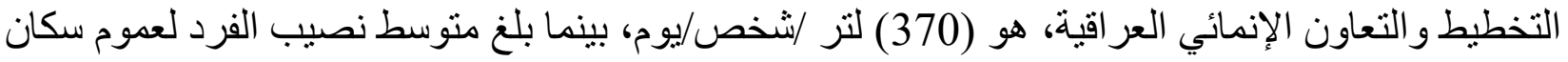

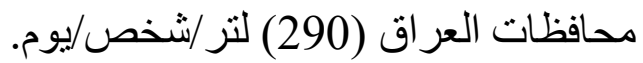

ان نسبة عدم رضا السكان عن خدمة توفير الماء بلغت (78\%) في مدينة العزيزية من مجموع العوائل، وبلغت

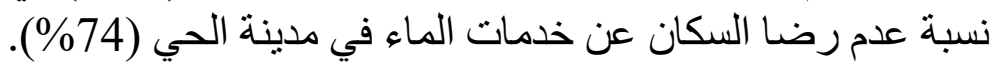

\section{مشكلات الصرف الصحي}

تعاني المدينتان من مشكلة عدم خدمات المجاري، فهي لا توجد إلاّ في بعض الأَحياء السكنية من مدينتي الحي

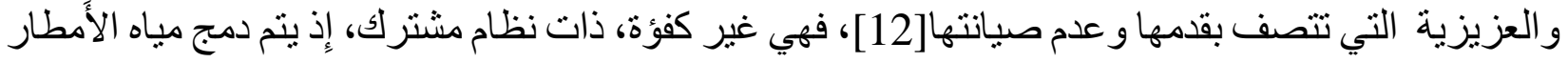

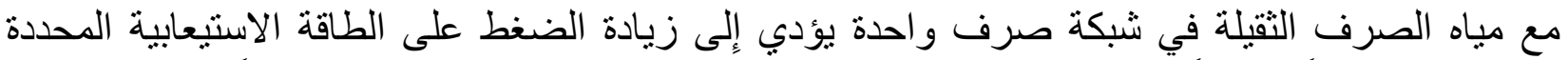

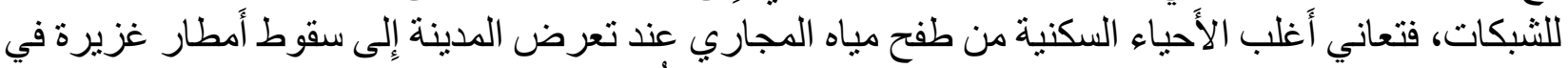

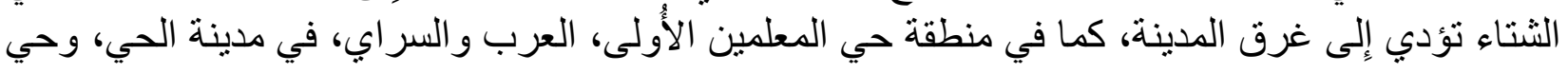

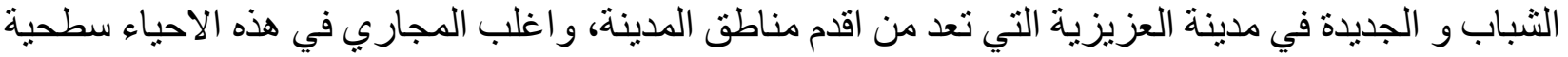

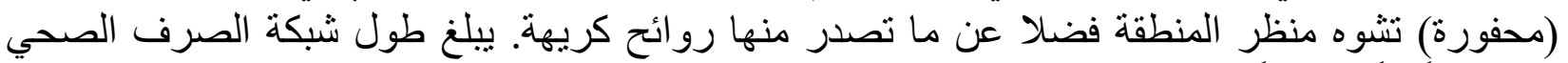

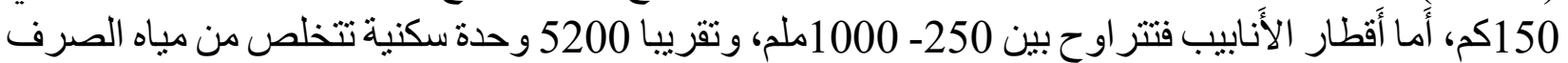
الصحي عن طريق الخزان الارضي، توجد محطة معالجة للصرف الصحي قيد التنفيذ تغطي 95\% من مدئئ مدينة

توجد شبكة تصريف المياه في اغلب مناطق العزيزية القديمة وهي ذات نظام مشترك (متداخل) الصرف الصحي

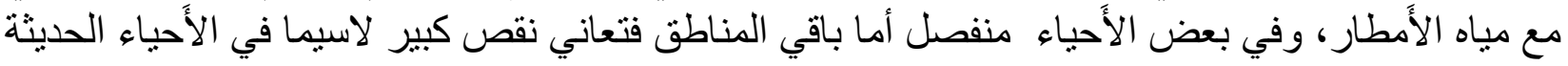
(الموظفين و السياسيين).

بلغت نسبة عدم رضا العو ائل عن خدمات المجاري عن طريق الدر اسة التي أُجريت (65\%) في مدينة العزيزية، وبنسبة (74\%) في مدينة الحي.

يتغذى قطاع كهرباء الحي من محطات ثانوية 11/33 كفا ثنانية ومحطات متنقلة عدد 4 جهد 11/132، مهنة وهي

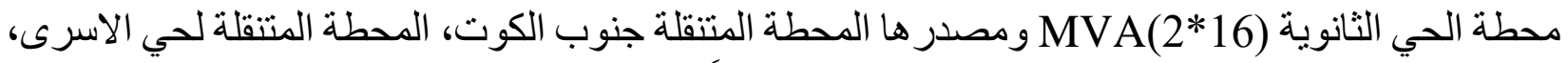
المحطة المتنقلة لناحية البشائر، محطة المجمع السكني، وأَنّ قدم المحطة الكهربائية في الحي و عدم مناسبة

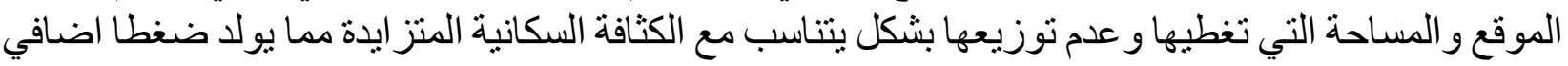
على الطاقة المتاحة، بسبب عدم وجود اعداد مناسبة من محطات التوزيع والتحويل فإن المناطق البعيدة تصلها 
طاقة كهربائية ضعيفة لا تشغل معظم اجز اء المنزل، دفع السكان الى شر اء المولدات الكهربائية المنزلية بأسعار

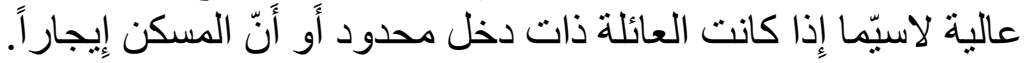
تعاني المدينة من مشكلة أُخرى هي مشكلة هي تدلي الاسلاك الكهربائية على البيوت و الشوار ع فيؤدي إلى تشوه منظر الثوارع ويعرض السكان للخطر ونلاحظ هذه المشكلة في حي العرب و السراي لكوني الكونها منطقة تجارية

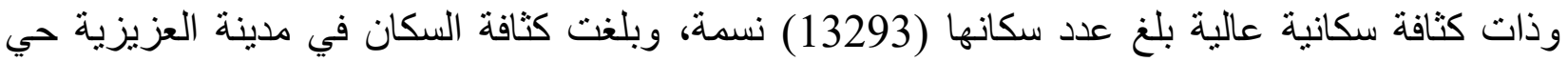
السعدونية والسر اي(7635) نسمة لذلك ز اد الطلب على الكهرباء من المولدات الاهلية (لهلية.

تستمد مدينة العزيزية حاجتها من الطاقة الكهربائية من محطة العزيزية (132/33/11) كيلوفولت التي تقع

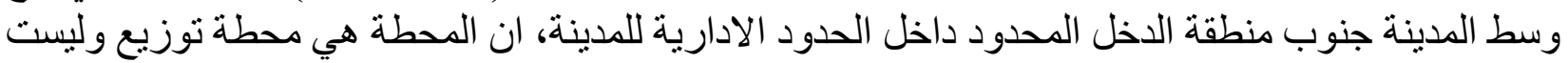

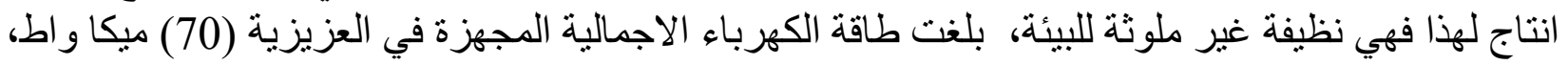

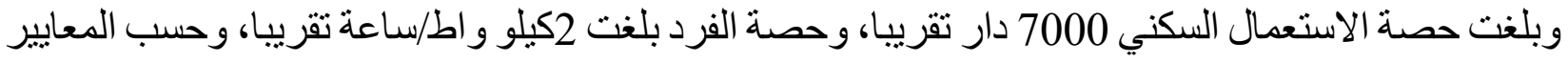
من وزارة التخطيط ان تبلغ حصة الفرد (422 ميكا و اط/شخص) يعني هذا وجود نقص كبير في الطاقة الكهربائية المجزة لمدينة العزيزية، ويتطلب هذا أنشاء مشاريع ومحطات لتوليد الطاقة الكائة الكهربائية لسد النقص

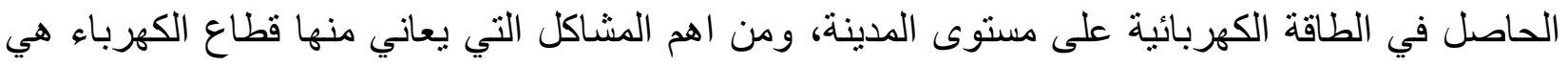

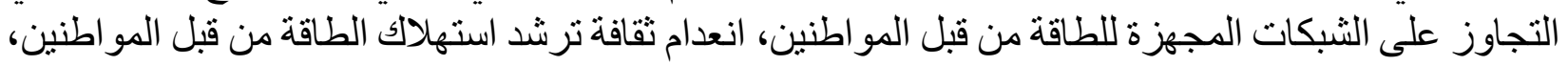

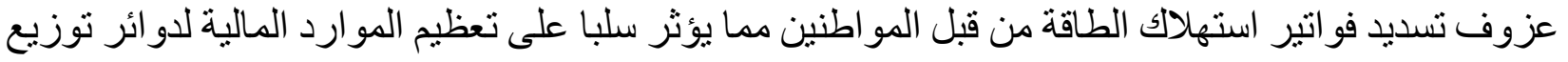

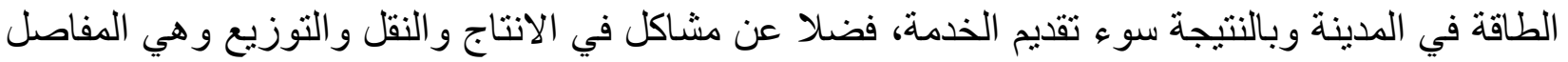
الثلاث التي يتمثل فيها مجال الطاقة الكهربائية.

و اتضح من المسح الميداني أَنّ (14\%) من عيَّنة الدراسة ابدوا رضاهم عن خدمة نوفير الطاقة الكهربائية،

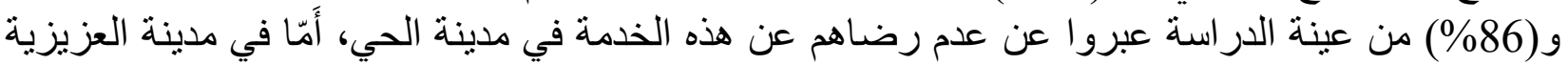

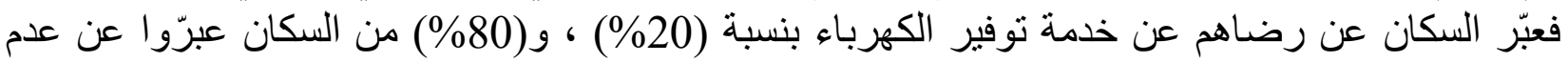
رضاهم عن هذه الخدمة .

\section{تناقص المساحات الخضراء المخصصة للترفيه}

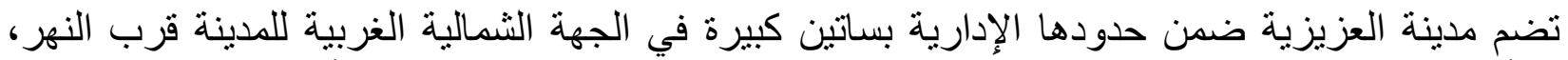

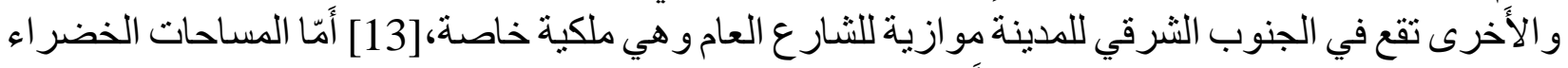

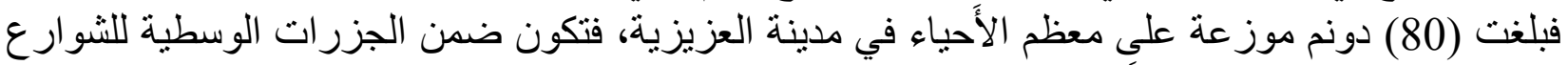

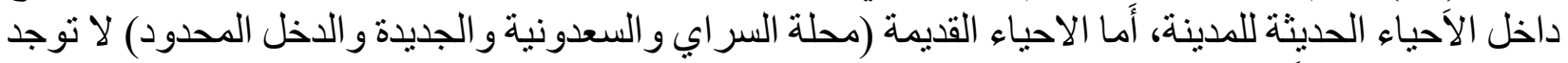

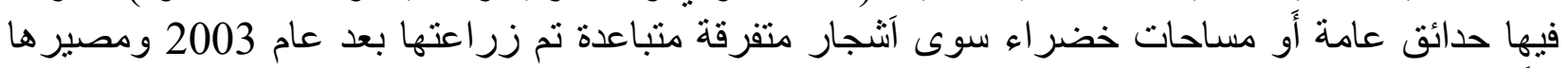

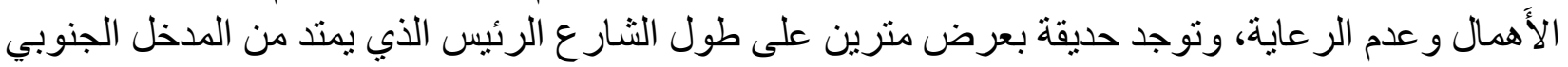

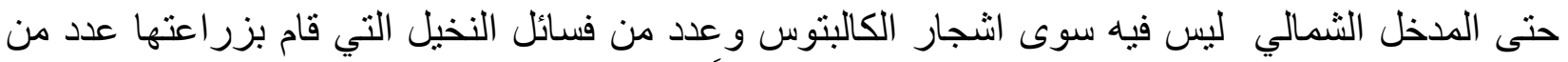

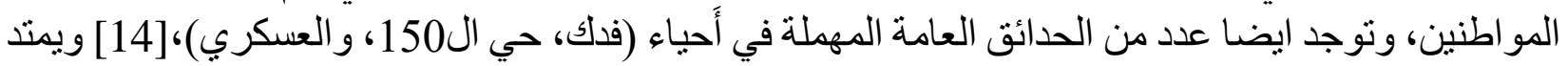

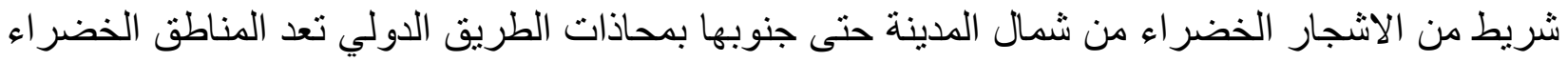

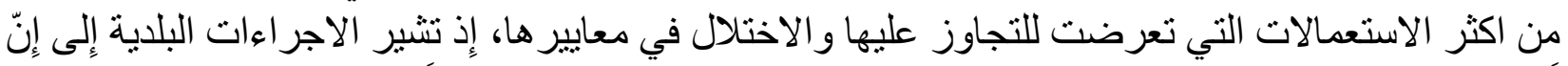

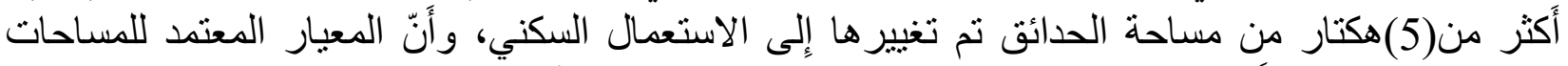

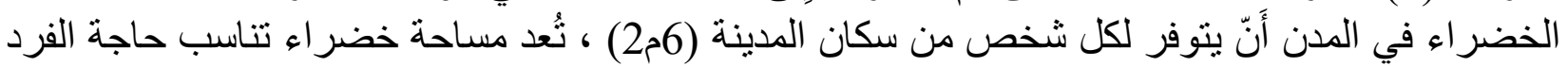
الترويحية والاجتماعية و النفسية و البيئية، و عند ضرب التب عدد سكان منطقة الدر اسة البالغ (58856) نسمة في (6م2) فِإن الحاجة الفعلية للمناطق الخضر اء يجب أَّْ تكون (353136م2) في مدينة العزيزية.

أَمّا في مدينة الحي فلا توجد مساحات خضر اء في المناطق القديمة بسبب عشو ائية السكن و عدم وجود تخطبط

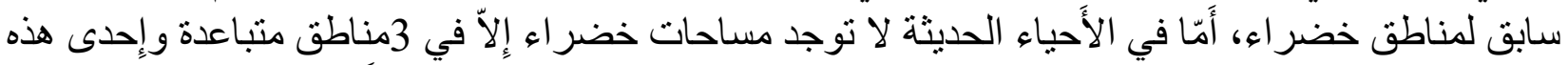

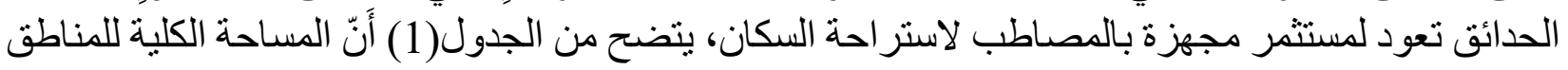




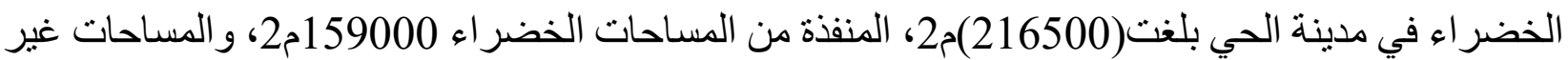

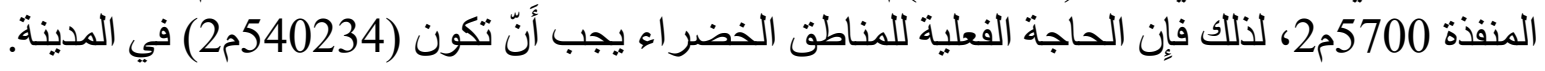

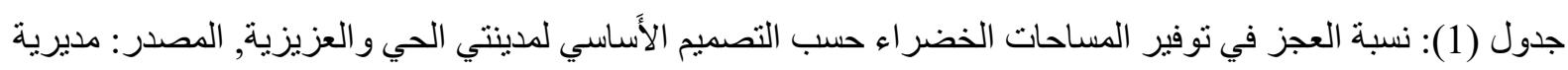

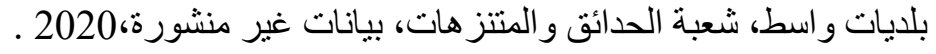

\begin{tabular}{|c|c|c|c|c|c|c|c|c|}
\hline المنبة العزاتز & النسبة العزات & الحعيار التخطيطي حسب & 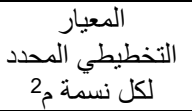 & عدد سكان & المبراحات المنفة م2 & $\begin{array}{l}\text { الخضاحاء } \\
\text { المنفذة م2 } \\
\text { المساتة }\end{array}$ & 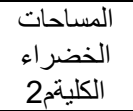 & المدينة \\
\hline \%72.8 & $\% 63$ & ${ }^{2}, 585254$ & $2 \uparrow 6.5$ & 90039 & 57000 & 159000 & 216500 & الحي \\
\hline$\% 61.9$ & $\% 51.2$ & ${ }^{2} 382564$ & $2 ; 6.5$ & 58856 & 40803 & 145715 & 186518 & العزيزية \\
\hline
\end{tabular}

\section{تراكم النفايات}

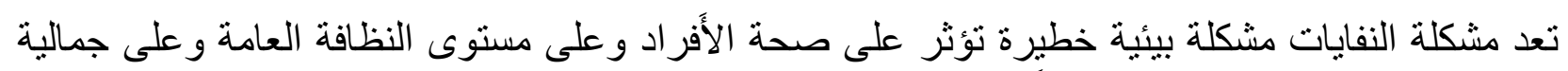

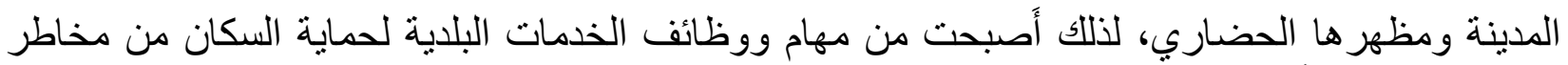

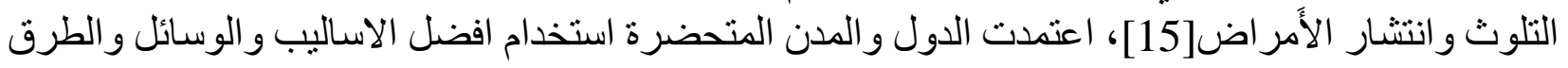

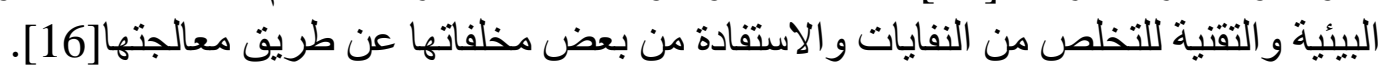

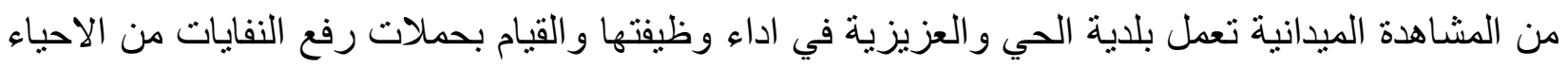

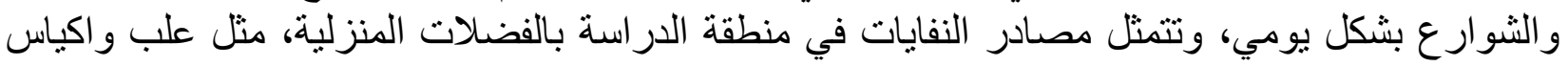

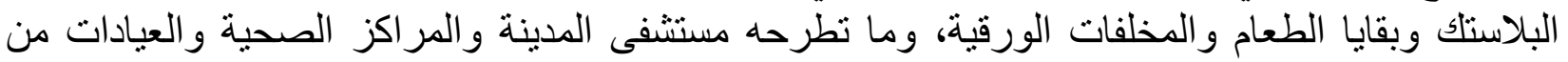

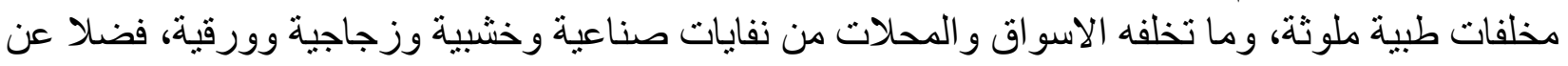
المخلفات الزر اعية التي تنجم عن الحدائق العامة و الحدائق المنزلية، كالحشائش والقات القش و الأشجار. في حين بلغت كمية النفايات المطروحة سنويا من قبل سكان مدينة العزيزية 24160طنة العناسنة ومعدل الكمية

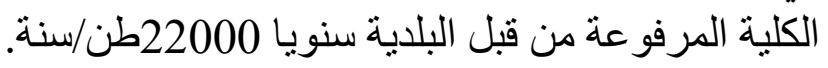

لقد تم تقدير ما بطرحه الفرد الواحد من النفايات الصلبة وفق المعايير التخطيطية والدحلية السائدة

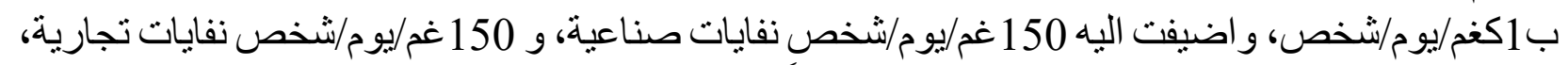

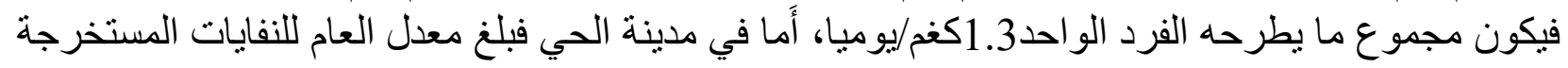

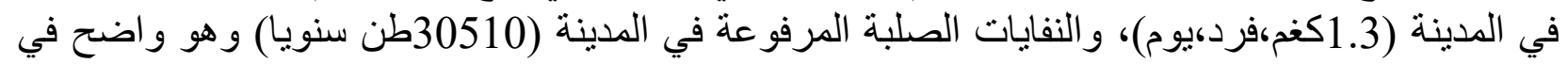

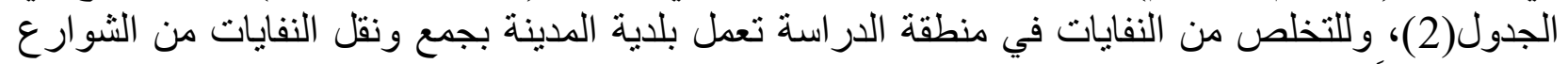

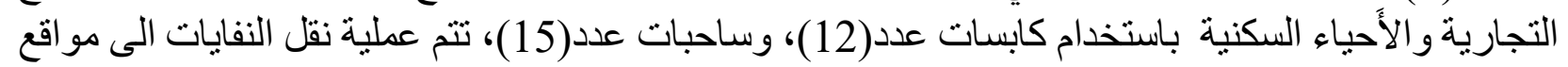

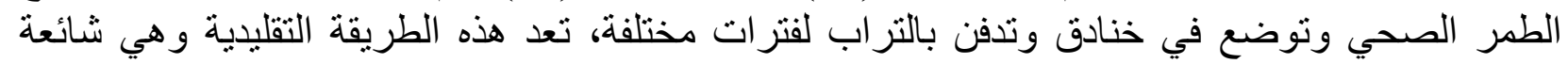

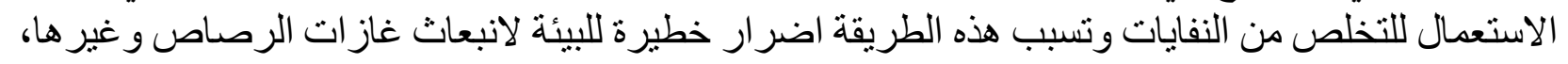

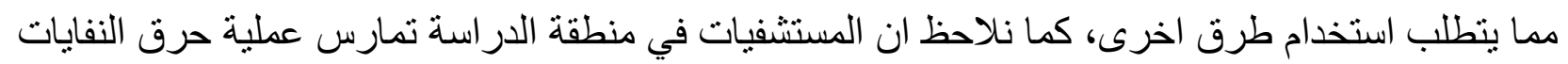

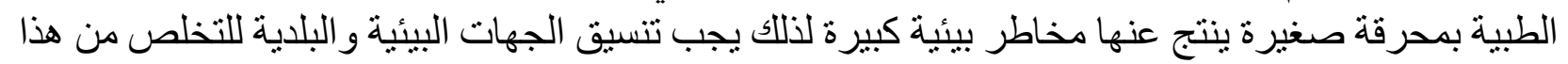

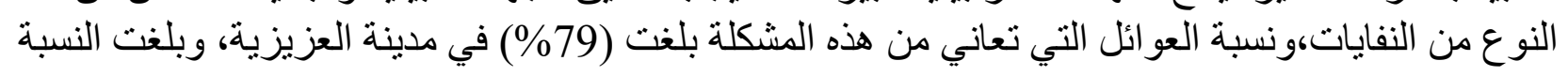
في مدينة الحي (855\%).

جدول (2): كمية النفايات المطروحة في مدينتي الحي ولعزيزية لعام 2020, الدصدر: مديرية بلديات واسط، شعبة البيئة،

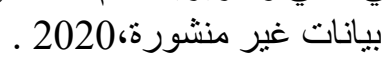

(*) تم تقدير الحاجة الفعلية من عدد السكان × المعيار التخطيطي.

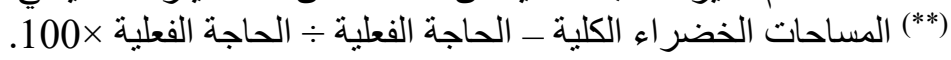

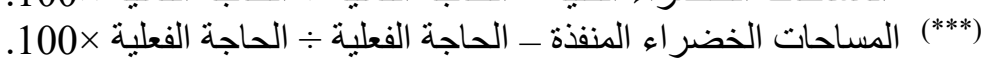




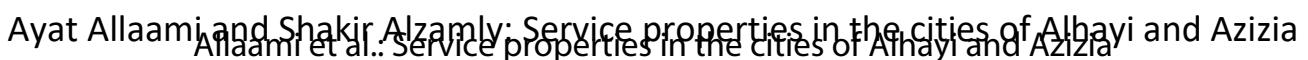

\begin{tabular}{|c|c|c|c|c|c|c|}
\hline كمية النفايات المرفوعة & كمية النفايات الصلبة المطروحة & كمية النفايات الصلبة المطروحة يوم & عدد نفوس المدينة & كمية الطرح للفرد كغم/ & 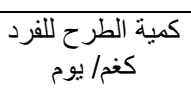 & المدينة \\
\hline 30510 & 42749.129 & 117.121 & 90039 & 474.5 & 1.3 & الحي ل الحي \\
\hline 27000 & 27833.221 & 76.255 & 58856 & 474.5 & 1.3 & العزيزية \\
\hline
\end{tabular}

1. تعاني حي الحرية من شـحة المياه في الصـيف بسـبب قدم الانابيب الناقلة للمياه و عدم كفاءتها اثر

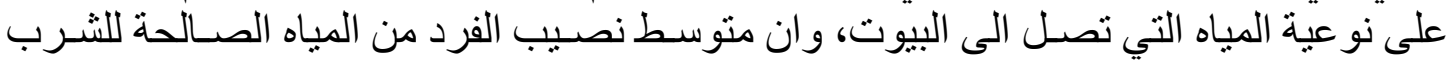

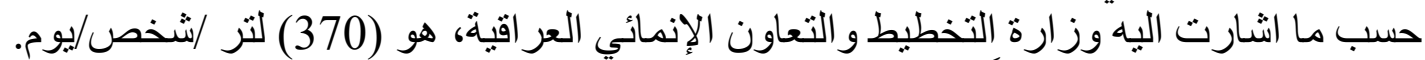

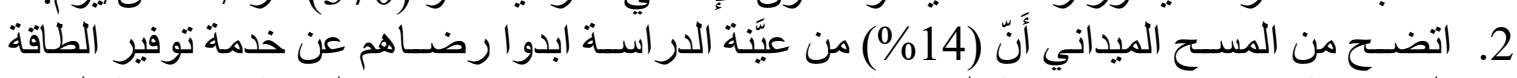

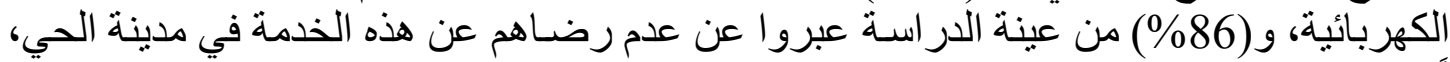

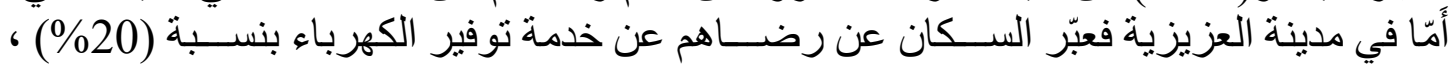

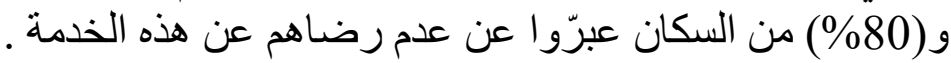

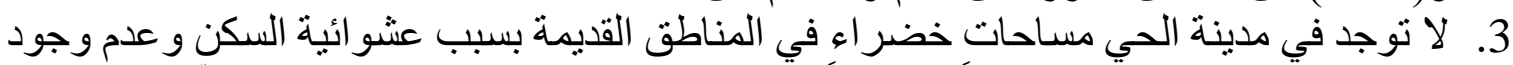

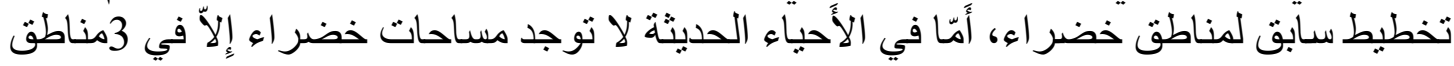

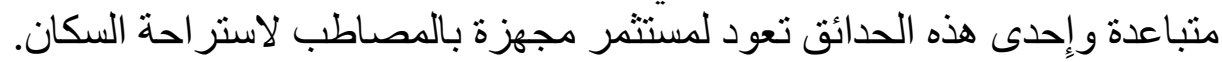

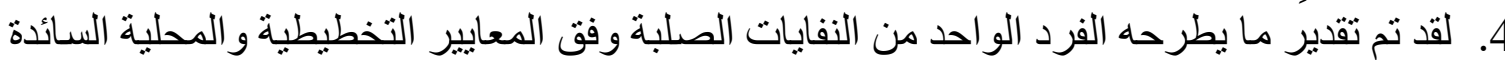

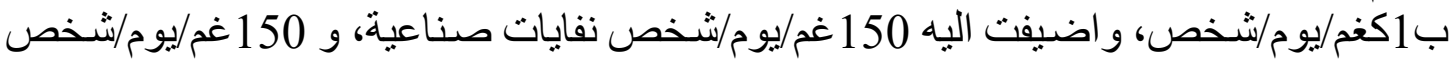

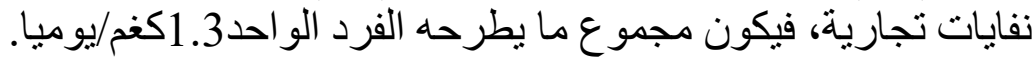

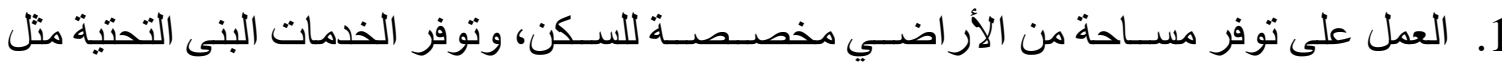

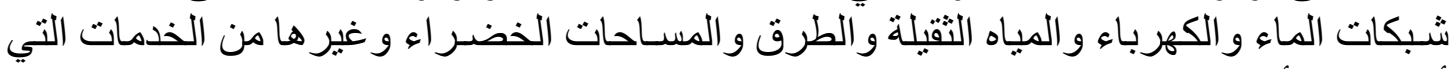

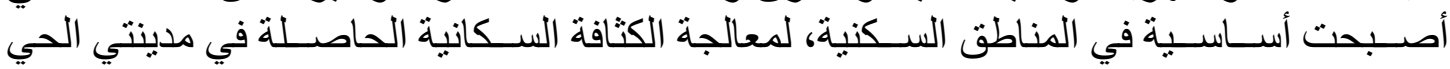
و العزيزية.

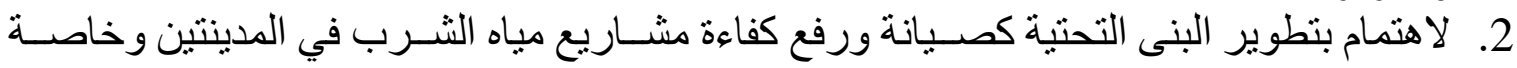

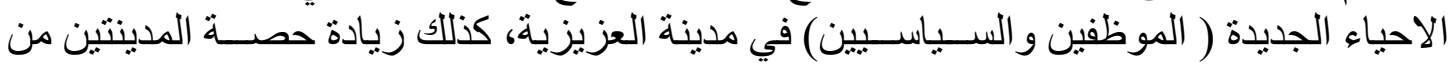

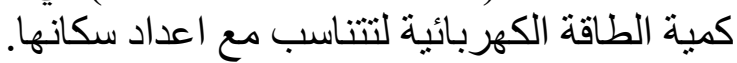

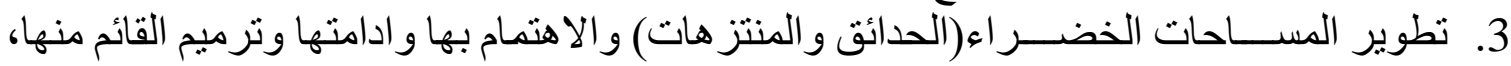

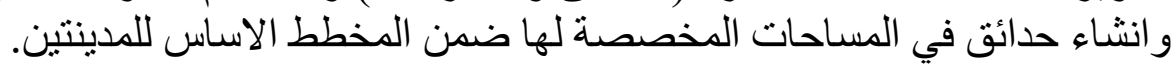

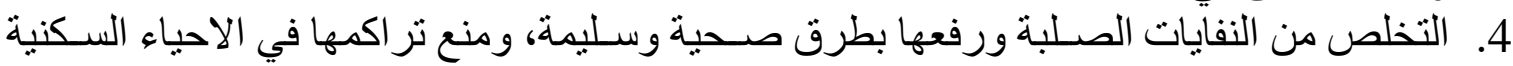
لغرض حماية هذه الاحياء و الحفاظ على البيئة السكنية من التلوثة

المصادر

[1] S. L. Farhan, "Sustainable neighborhood Comparative Analysis of Al Kut Neighborhoods Sustainable neighborhood Comparative Analysis of Al Kut Neighborhoods," no. December, pp. 302-317, 2018.

[2] I. Abbass Jasim, S. Lafta Farhan, and S. AL-MAMOORI, "Smart Government: Analysis of Shift Methods in Municipal Services Delivery: The Study Area: Al-Kut - Iraq,” J. Univ. Kerbala, vol. 15, no. 3, p. 2017, 2017. 
[3] A. N. Sabeeh Lafta Farhan, Ihsan Abbass Jasim, "Urban sustainability in Old City Centres, a Comparison Between the City of Najaf in Iraq and Italian Cities Experiences," no. 12, 2016.

[4] I. A. Jasim, S. L. Farhan, and H. M. Hasan, "Ways to Activate Urban Transport to Achieve Urban Sustainability," IOP Conf. Ser. Mater. Sci. Eng., vol. 1090, no. 1, p. 012034, 2021.

[5] S. Farhan and M. G. Abdelmonem, "Navigating the socio-spatial and planning conditions of traditional public spaces in Iraq's holy cities," in Traditional Dwellings and Settlements Review, 2018, vol. 30, no. 1, p. 81.

[6] I. A. Jasim, H. M. Hasan, S. L. Farhan, and K. H. Bahat, "Evaluating the urban structure of Al-Kut city according to sustainability," \{IOP $\}$ Conf. Ser. Earth Environ. Sci., vol. 779, no. 1, p. 12021, Jun. 2021.

[7] I. A. Jasim, S. L. Farhan, and H. M. Hasan, "The Impact of Transit on Sustainable Urban Form,” IOP Conf. Ser. Mater. Sci. Eng., vol. 1058, no. 1, p. 12049, 2021.

[8] S. L. Farhan, H. H. Samir, and H. S. Adelphi, "Urban changes and its impact on the tangible and intangible heritage of City's Centre: Najaf City as a Case Study," IOP Conf. Ser. Mater. Sci. Eng., vol. 1058, no. 1, p. 12070, 2021.

[9] S. lafta farhan, "Deterioration in the centers of historic towns The case study: the residential environment for the ancient of Kut city," iraqi J. Archit. Plan., vol. 10, no. 3, pp. 128-148, 2014.

[10] H. A. et al. S. Farhan, T.Mutas, "Digital Era Influence on Neighbourhood Planning," I O P Conf. Sci. Mater., 2021.

[11] sabih 1. Farhan, "Visual pollution in the commercial urban scene Analysis of the optical axes elected in the Kut city.," Wasit J. Eng. Sci., no. 4, pp. 178-208, 2016.

[12] H. S. O. Adelphi, S. L. Farhan, and H. A. H. Alshamari, "The Threshold of Urban Sustainability within the Traditional Cities: Traditional Alnajaf city as a case study," \{IOP\} Conf. Ser. Mater. Sci. Eng., vol. 1058, no. 1, p. 12055, Feb. 2021.

[13] S. L. Farhan, H. I. Alyasari, V. S. Akef, S. L. Zubaidi, and K. S. Hashim, “Analysing the Transformed Urban Patterns of Al-Najaf Historical Center: Urgent Issues and Possible Solutions," IOP Conf. Ser. Mater. Sci. Eng., vol. 1058, no. 1, p. 12052, 2021.

[14] S. L. Farhan, H. I. Alyasari, H. H. Samir, S. L. Zubaidi, and K. S. Hashim, "Conservation Approach as an Architectural Instrument to reviving Historical Cities; technical analysis for multi international cases," IOP Conf. Ser. Mater. Sci. Eng., vol. 1058, no. 1, p. 12071, 2021.

[15] S. L. Farhan, "Effect of New Urban Transformations at Townscape of Historical Cities Urban Study of Alnajaf Hist," J. Eng., vol. 23, no. December, pp. 1-20, 2018.

[16] S. L. Farhan, V. S. Akef, and Z. Nasar, "Revitalizing the historical center of Al-Najaf city in Iraq: learning from the British conservation experiences," J. Cult. Herit. Manag. Sustain. Dev., vol. ahead-of-p, no. ahead-of-print, Jan. 2021.

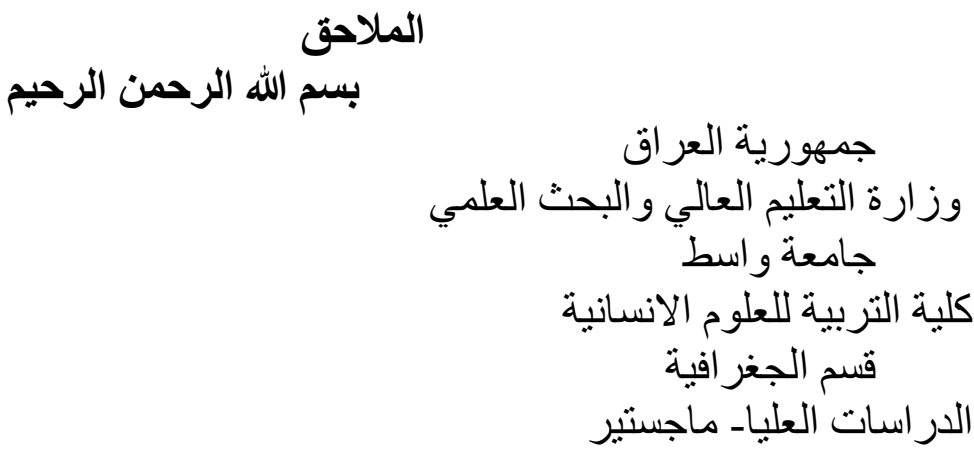




\section{استمارة المسح الميداني للبحث الموسوم \\ ( (الحاجة السكنية لمدينتي الحي والعزيزية دراسة مقارنة ) )}

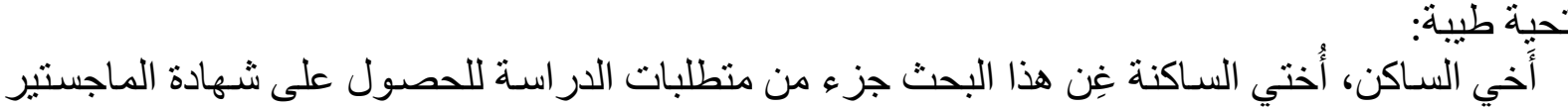

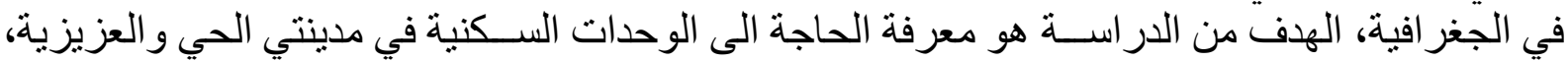

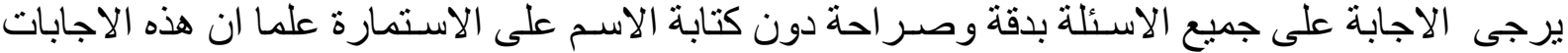

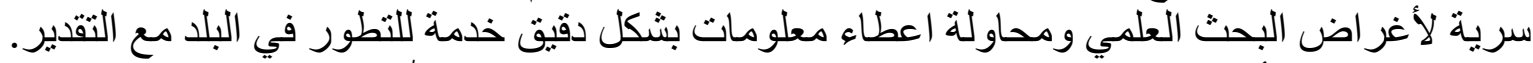

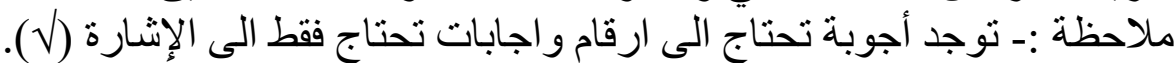

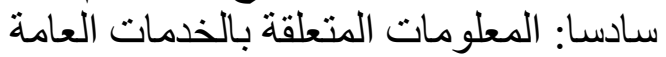

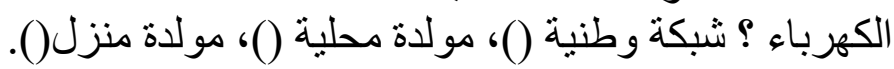

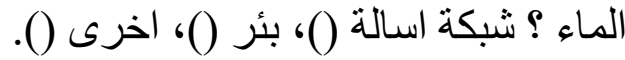

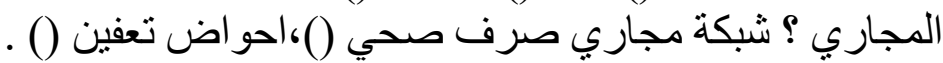

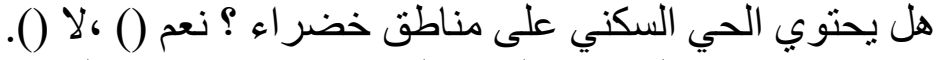

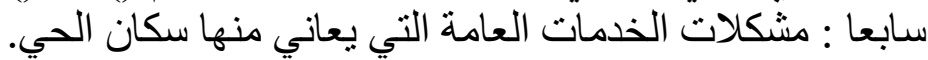

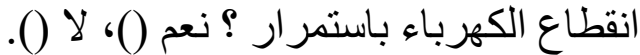

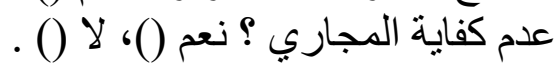

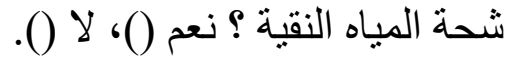
تر اكم النفايات ؟ نعم ()، لا () . (). 